\title{
RADIO-FREQUENCY MULTI-MODE MICROMECHANICAL DISK RESONATORS IN 500nm THIN SILICON CARBIDE (SiC)
}

\author{
H. Zamani ${ }^{*}$, J. Lee ${ }^{*}$, S. Rajgopal, C.A. Zorman, M. Mehregany, and P.X.-L. Feng ${ }^{\dagger}$ \\ Electrical Engineering, Case Western Reserve University, Cleveland, OH 44106, USA \\ *Equally contributed authors. ${ }^{\dagger}$ Email: philip.feng@case.edu
}

\begin{abstract}
We report on experimental demonstration of a new type of radio-frequency (RF) multi-mode micromechanical resonators based upon vibrating circular disks made of a 500nm thin $\mathrm{SiC}$ epilayer grown on single crystal $\mathrm{Si}$. These center-clamped microdisk resonators offer multiple flexural-mode resonances at $\mathrm{RF} /$ microwave frequencies, depending on both disk and anchor diameters. We show that simply changing the anchor size can already tune the interesting patterns of the multi-mode frequency spectra. A $\sim 40 \mu \mathrm{m}$-diameter $\mathrm{SiC}$ disk with a slender ( 800nm) anchor exhibits more than a dozen flexural modes in $\sim 2-20 \mathrm{MHz}$ with typical quality factors $Q$ 's $\sim 1000-4000$. A disk with same dimensions but a wide $(\sim 20 \mu \mathrm{m})$ anchor has its first 9 flexural modes within $15-20 \mathrm{MHz}$ with $Q$ 's $\sim 500-2500$. The measurements and analyses show that the frequency spacing between the flexural modes, the splitting of degenerate modes, and their $Q$ 's (or dissipation), can all be tuned by engineering the size and shape of the anchor of a multi-mode flexural thin-film microdisk resonator.
\end{abstract}

\section{INTRODUCTION}

Mechanical disk structures (e.g., rectangular or circular) offer versatile resonance modes due to their many interesting vibrations ranging from flexural (transverse, bending) to bulk (extensional, contour), and also dependent upon their geometries and boundary conditions. Such resonances have hence been widely exploited for signal processing and resonant sensing applications [1,2]. Recent advances in microelectromechanical systems (MEMS) have led to various high- $Q$ microdisk resonators $[3,4]$, in which contour, extensional and other bulk, high-stiffness modes of thick disks are advantageous toward RF/microwave frequency control and timing applications. In today's nanoelectromechanical systems (NEMS) enabled by many emerging nanostructures such as nanowires/tubes and nanobeams/cantilevers, flexural motions (bending, deflection) are prevailing; and the available high- $Q$ flexural resonances are often advantageous for dynamical sensing of ultra-small quantities and effects [5], and for making low voltage and ultra-low power $\mathrm{RF} /$ microwave NEMS [6]. $\mathrm{SiC}$ is an attractive material and proven to be excellent for NEMS toward these goals [5-8], thanks to its exceptional electromechanical properties and nanomanufacturing availability in very thin layers on various sacrificial materials.

In this work, we take a very thin singly-clamped SiC NEMS cantilever, and envisage it to rotate in plane around its clamp and thus extrude into a thin, circular disk (Fig. 1). This new disk is intriguing from both NEMS and MEMS view points. It is much thinner than mainstream bulk-mode MEMS disk resonators; and it has an extremely large surface area in contrast to its original cantilever template (hence a tremendous enhancement on available active surface for capturing, critical for sensing applications). Moreover, the disk derived from the SiC NEMS cantilever would be only anchored and supported by a post/pillar underneath at the center. Here we describe the experimental implementation of this idea, including a novel fabrication process for making the very thin $\mathrm{SiC}$ microdisks, measurement of the $\mathrm{SiC}$ disks' flexural-mode resonance response, and the $\mathrm{SiC}$ disks' multi-mode operations. We focus on exploring the multi-mode characteristics and the effects of varying anchor size upon device performance.

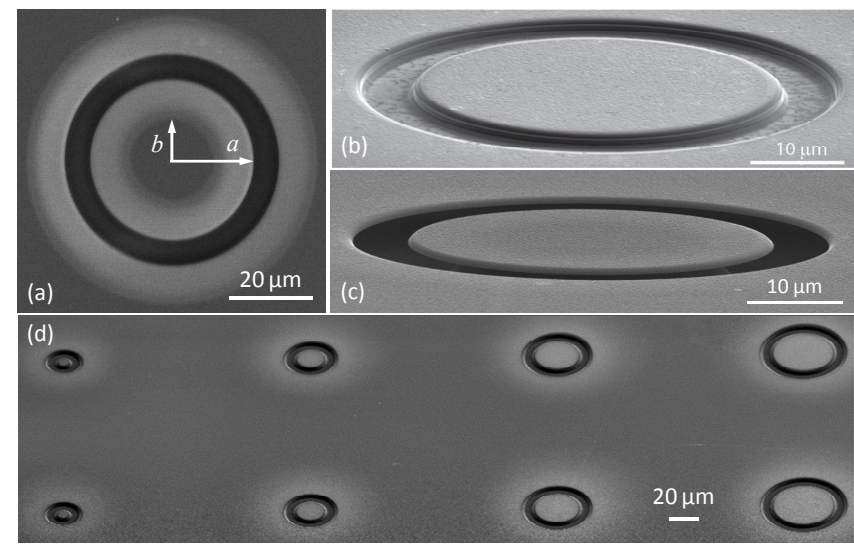

Figure 1: Examples of prototyped 500nm thin SiC circular microdisk resonators and arrays. (a) Top-view scanning electron

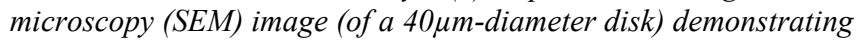
the size of the microdisk (radius a) and the underneath supporting pillar (radius b). (b) \& (c) Titled-view $\left(30^{\circ} \& 75^{\circ}\right.$ respectively) SEM images of the same device before and after Si etch for device suspension. (d) An array of microdisks with various diameters.

\section{DEVICE FABRICATION}

The 500nm thin SiC epilayer is heteroepitaxially grown on single crystal Si (100) by an atmospheric pressure chemical vapor deposition (APCVD) process [9]. As shown in Fig. 2, the device nanomachining process features a simple mask-less design and a resist-less protocol. First, circular rings of width $\sim 5-10 \mu \mathrm{m}$ are directly patterned and nanomachined on the $\mathrm{SiC}$ epilayer, passing the $\mathrm{SiC} / \mathrm{Si}$ interface, by using focused ion beam (FIB) (Figs. $1 \& 2$, specifically, for majority of the devices, with $30 \mathrm{keV}, 47 \mathrm{nA}$, on a state-of-the-art FEI Helios NanoLab 650). The exposed Si is then etched in HNA for various times (depending on desired sizes of the anchoring pillars for the disk resonators) according to calibrated etching rates in this specific process. We prove this to be an excellent protocol in enabling various designs and fast prototyping and testing of thin-film SiC microdisk resonators and arrays.

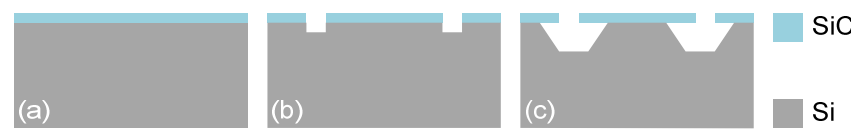

Figure 2: Process flow for fabrication and prototyping of $\mathrm{SiC}$ microdisks. (a) 500nm 3C-SiC epilayer on Si (100) substrate. (b) Mask-less patterning and nanomachining of SiC using FIB. (c) Etching of Si substrate by HNA to yield suspended SiC microdisks.

\section{MICRODISK RESONANCE MEASUREMENT}

The suspended microdisks are tested all at room temperature in a vacuum of $\sim 12 \mathrm{mT}$ Torr to examine their resonance response, by using a home-built apparatus [10] that incorporates a two-port RF network analysis measurement with a sensitive laser interferometry for displacement readout. $\mathrm{SiC}$ microdisk samples are driven by a piezoelectric thin disk actuator. Every observed micromechanical resonance is examined and carefully verified to be from the vibrational modes of the SiC microdisk. To ensure this, we have 
employed a combination of techniques and tests, which generally include examinations of amplitude dependency, linear and nonlinear behaviors at varying drive levels, precise response dependency on laser spot locations on the device surface, control tests of response and dependency when the same laser spots focused upon areas with no devices (Fig. 1d, regions between microdisks), careful calibration and tuning of the spot size (and other specs) of the laser interferometry, dependency on varying laser intensity, examination of thermomechanical fluctuations (Brownian motions) of micromechanical devices, etc. For each device, after convincingly verifying the observed micromechanical modes, the optical detection is further optimized to measure the overall response for each device. The detection is then tuned and optimized for characterizing individual peaks and modes.

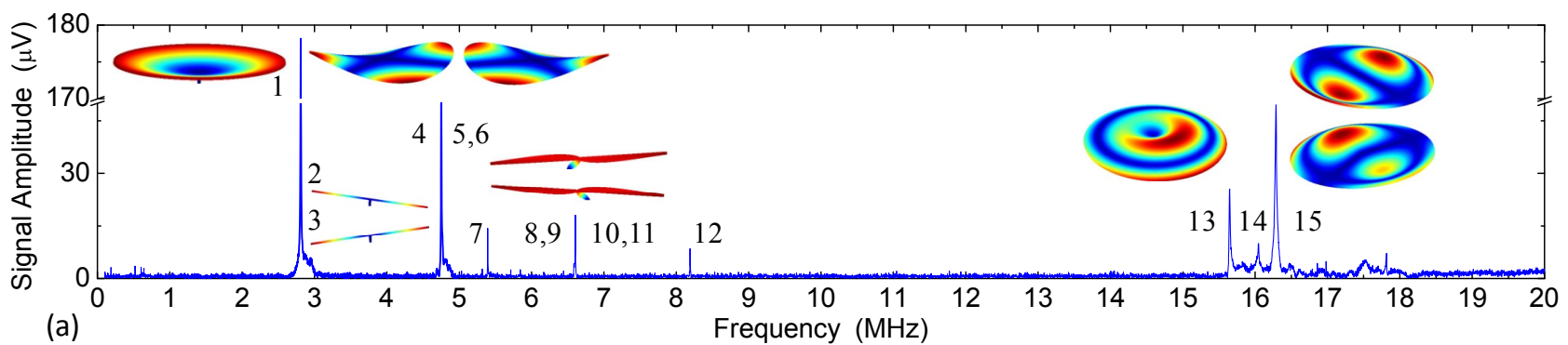

(a)
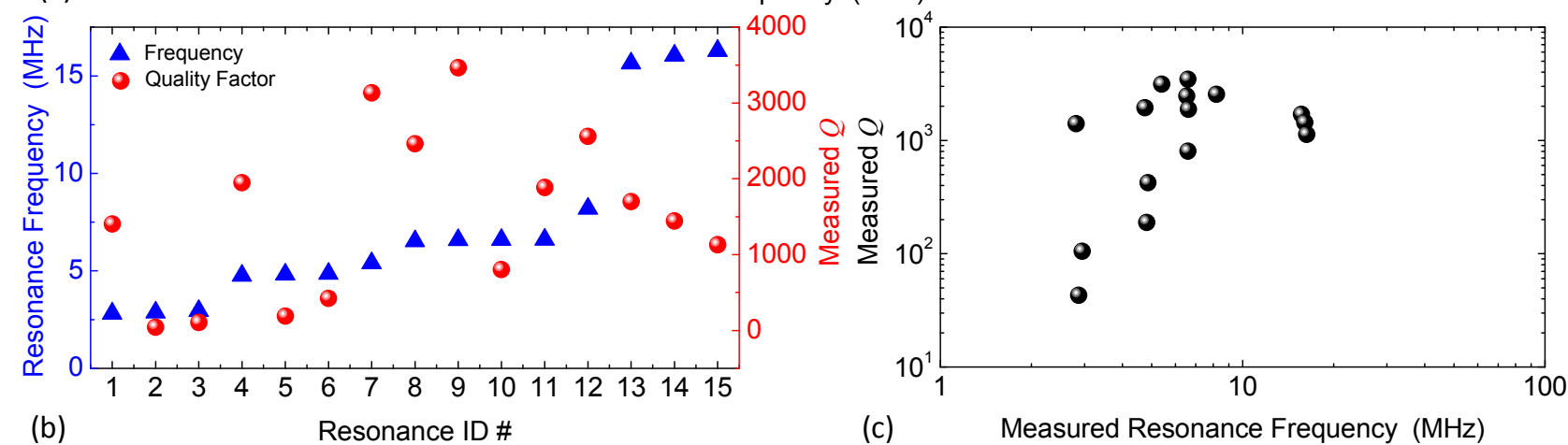

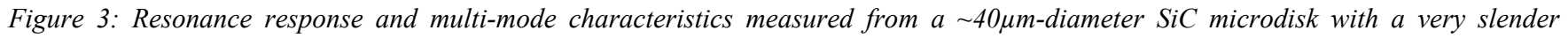
anchoring stem (diameter $\approx 800 \mathrm{~nm}$ ). (a) Wide range $R F$ multi-mode response measured by using two-port RF network analysis with laser interferometric detection techniques. A resonance ID \# is designated to each observed and carefully verified micromechanical resonance, in the order of increasing frequency. Insets: simulated mode shapes. (b) Measured resonance frequency (triangles) and $Q$ 's (circles) versus resonance ID \#. (c) Measured $Q$ versus resonance frequency with a trend defying the conventional $Q$-frequency trade-off.

\section{EXPRIMENTAL RESULTS AND DISCUSSIONS}

\section{SiC Microdisk Resonators with Slender Anchors}

Figure 3 shows the main results of measured flexural response from a 500nm-thick, $\sim 40 \mu \mathrm{m}$-diameter SiC disk with an anchoring stem of only $\sim 800 \mathrm{~nm}$ in diameter. All the verified resonance peaks are from out-of-plane vibrations and a few modes that involve the flexural motions of the thin disk and the slender stem together. Analytical and finite element (COMSOL) modeling are combined to analyze and determine the mode shapes, as illustrated in Fig. 3a.

The interesting step-like pattern of the frequency versus mode number in Fig. $3 b$ clearly indicates the predicted degenerate modes (almost overlapping frequencies) due to the disk's many symmetry possibilities (with abundant theoretical nodal diameters and nodal circles). Structural and fabrication nonidealities break degeneracy and cause mode splitting; hence many of the observed peaks, each in fact is a superposition of two split degenerate modes (Fig. 4b).
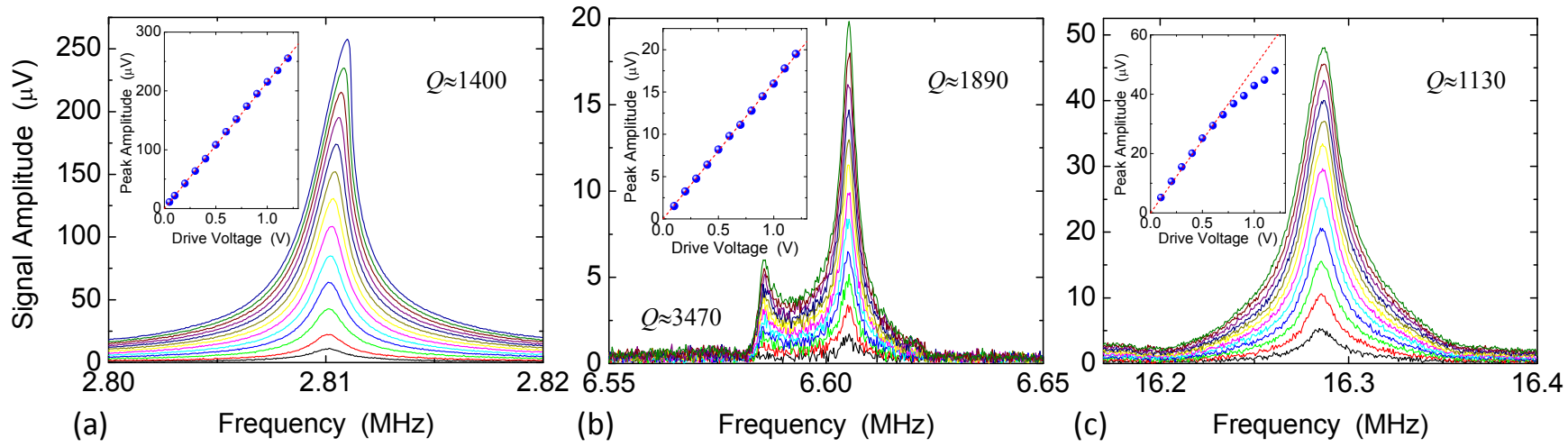

Figure 4: Characteristics of individual resonance modes measured from the SiC microdisk with a very slender supporting stem. (a) The fundamental out-of-plane flexural resonance at $\sim 2.81 \mathrm{MHz}$, with $Q \approx 1400$. (b) A pair of modes at $\sim 6.6 \mathrm{MHz}$ involving flexural vibrations of the slender stem. (c) A high frequency flexural mode at $\sim 16.3 \mathrm{MHz}$, with $Q \approx 1130$, showing nonlinear behavior at high amplitude. Insets: measured peak amplitude of the resonance (or the dominant resonance, in panel (b)) versus drive voltage, along with linear fitting. 

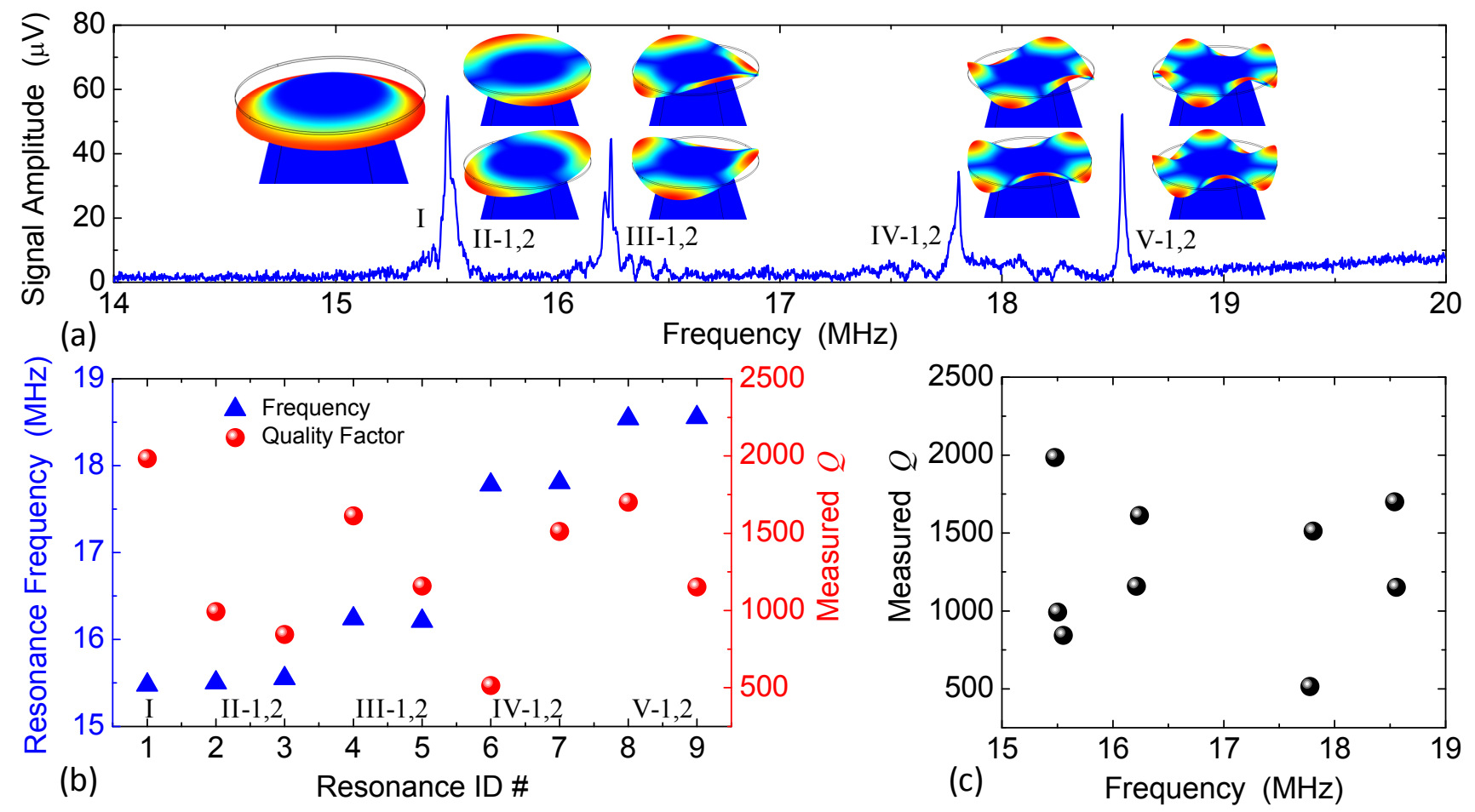

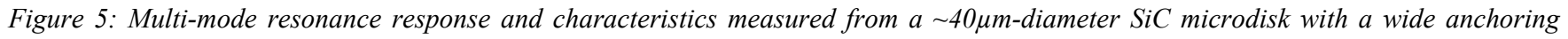
pillar (diameter $\approx 20.9 \mu \mathrm{m}$ ). (a) Wide range RF multi-mode response. The observed major flexural modes are designated I, II, III, IV \& $V$, and the splitting 'degenerate' modes are labeled $1 \& 2$, according to modeling predictions. Insets: simulated mode shapes. (b) Measured vibrational resonance frequency (triangles) and $Q$ (circles) versus resonance ID \#. (c) Measured $Q$ versus resonance frequency.

Figures $3 \mathrm{~b} \& \mathrm{c}$ show, very interestingly, a non-monotonic $Q$ frequency dependency and an overall trend of possibly overcoming the conventional $Q-f$ trade-off. Figure 4a shows the fundamental flexural resonance at $\sim 2.81 \mathrm{MHz}$ from the disk, with its simulated mode shape shown in Fig. 3a close to the peak. Figures $4 \mathrm{~b} \& \mathrm{c}$ demonstrate selected examples of observed mode-splitting, and transition from linear to nonlinear characteristics, respectively.

\section{SiC Microdisk Resonators with Wide Anchors}

When the anchor becomes as wide as $\sim 1 / 2$ of the disk diameter (the images of the specific device shown in Fig. 1a \& c), measurement results in Fig. 5 demonstrate that the first 9 measured flexural modes are all shifted to higher frequencies $(\sim 15-20 \mathrm{MHz}$, compared to results in Fig. 3). This is due to the shortened radial length of the suspended part (rim) of the disk, or corresponding to a shorter cantilever with a wider clamping port that would rotate and extrude into this circular disk.

A compelling step-like pattern of mode frequencies (with counts 1-2-2-2-2) in Fig. 5b clearly shows the degeneracy as predicted. The insets in Fig. 5a illustrate the ideal mode shapes, where each in the II, III, IV, and V pairs has 1, 2, 3, and 4 nodal diameters, respectively. In contrast to the previous device and results shown in Fig. 3, due to the much shorter suspended radial length, in this device the mode shapes with nodal circles will only occur at frequencies much higher than $20 \mathrm{MHz}$. Obviously, in this device the anchor is much more rigid (very high stiffness), and in the measured range, there is clearly no flexural mode that is coupled to either lateral or vertical motions of the anchor.

Figures $5 \mathrm{~b} \& \mathrm{c}$ show a non-monotonic $Q-f$ dependency, similar to that in Fig. 4. The overall trend, however, appears to be toward decreasing $Q$. This suggests clamping loss may be more important in higher frequency disks/modes with wider anchors.

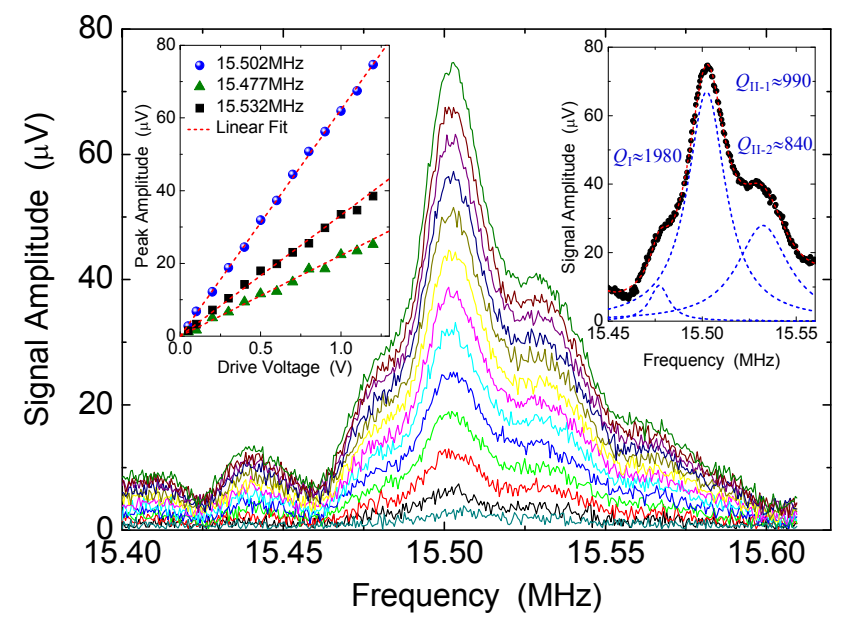

Figure 6: Characteristics of resonance modes at $\sim 15.5 \mathrm{MHz}$. Main plot shows response measured by sweeping $V_{d r v}=0.1-1.2 \mathrm{~V}$ with $0.1 \mathrm{~V}$ steps. Left Inset: Measured peak amplitude of the modes versus drive, with linear fits to the data. Right Inset: Analysis and decomposition of the measured response into three adjacent modes according to careful modeling, with extracted $Q$ values.

Nonidealities cause mode splitting near the ideal degenerate frequencies. Therefore each of the observed peaks II, III, IV, and $\mathrm{V}$ presents a superposition of a pair of split modes. Some nonideal effects not only cause frequency discrepancies, but also strongly change the split modes' amplitudes (e.g., a very small mismatch between the centers of anchor and disk can significantly change the relative amplitudes of the split pairs, and the color-mapped mode 
shapes will be quite different than shown in Fig. 5a). Therefore, while ideally the amplitudes of each pair of modes should be equal and mode shapes be symmetric (Fig. 5a), in fact one split mode can easily have a much higher amplitude response and dominate over the other. Given repeated observations of this imparity, we combine data and multi-Lorentzian analysis to decompose the measured peaks and extract the predicted split modes guided by modeling. Figures 6-9 clearly reveal the detailed characteristics (dominance $\&$ contributions in each pair) resolved by this analysis.

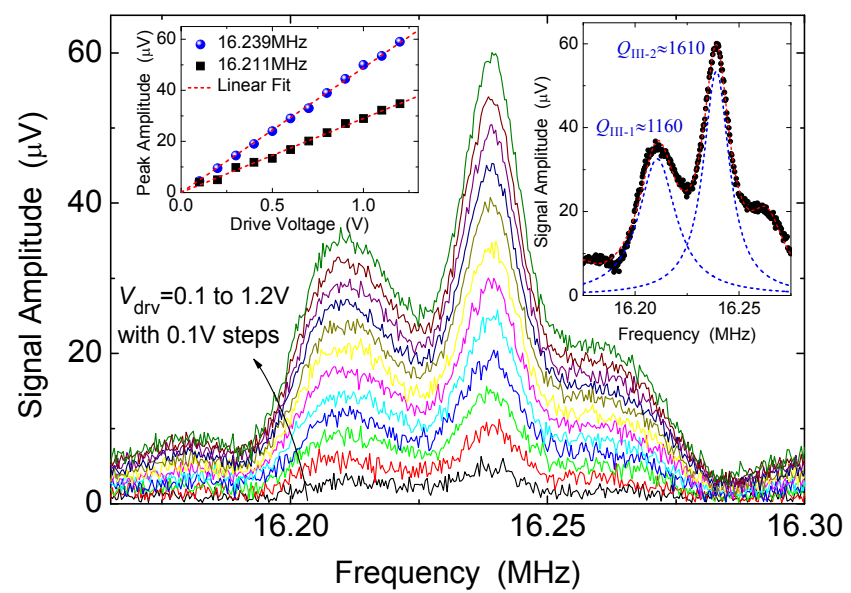

Figure 7: A pair of vibrational modes at $\sim 16.21 \mathrm{MHz}$ and 16.24MHz. Left Inset: Measured peak amplitude of the modes versus drive. Right Inset: Analysis of the contribution from the two split modes to the measured response, and the extracted $Q$ 's.

The very small mode splitting $\left(\Delta f_{N-1,2} / f_{N}<0.2 \%\right.$, for $\left.N=\mathrm{II}-\mathrm{V}\right)$ is just comparable or even smaller than the resonance peak width, further increasing the complications caused by amplitude imparity. While natural (nonidealities induced) mode splitting may help explore intrinsic issues in devices and materials, for specific device applications, sub-resonance-width natural mode splitting may need to be intentionally evaded by design with asymmetries.

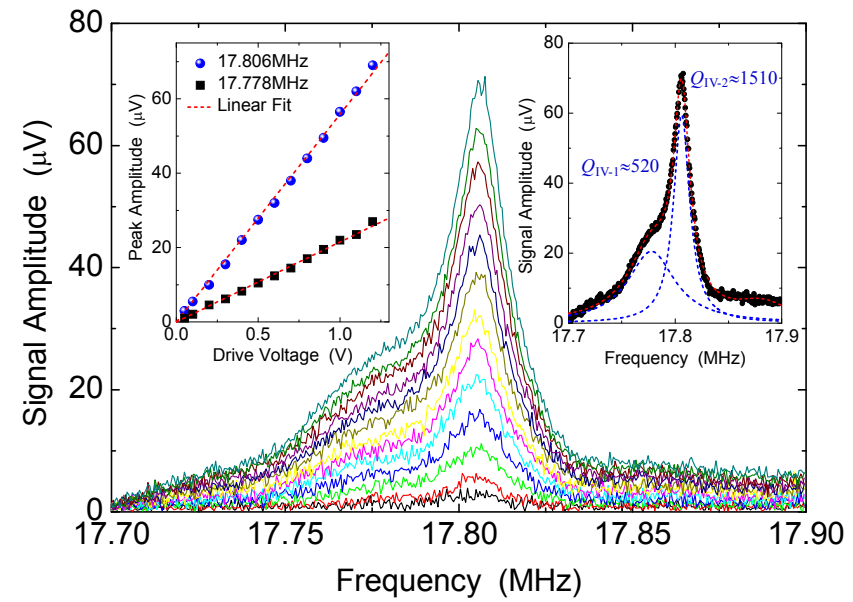

Figure 8: Measured response of an uneven pair of split modes at $\sim 17.78 \mathrm{MHz}$ and $\sim 17.81 \mathrm{MHz}$ (dominating). Left Inset: Peak amplitude versus drive with linear fits. Right Inset: Analysis and revelation of the two split modes, and the extracted $Q$ 's.

We note that the measured multi-mode response is very sensitive to the tuning of the laser interferometry and precise control of the laser spot on the surface of the SiC microdisks. Importantly, each of the full wide-range data traces in Fig. 3a and
Fig. 5a is taken without relocating the spot, simply by optimizing the overall full-range response only. A larger spot is used in obtaining the Fig. 3a data, while a smaller spot is focused on the suspended portion of the microdisk in case of the data in Fig. 5a.

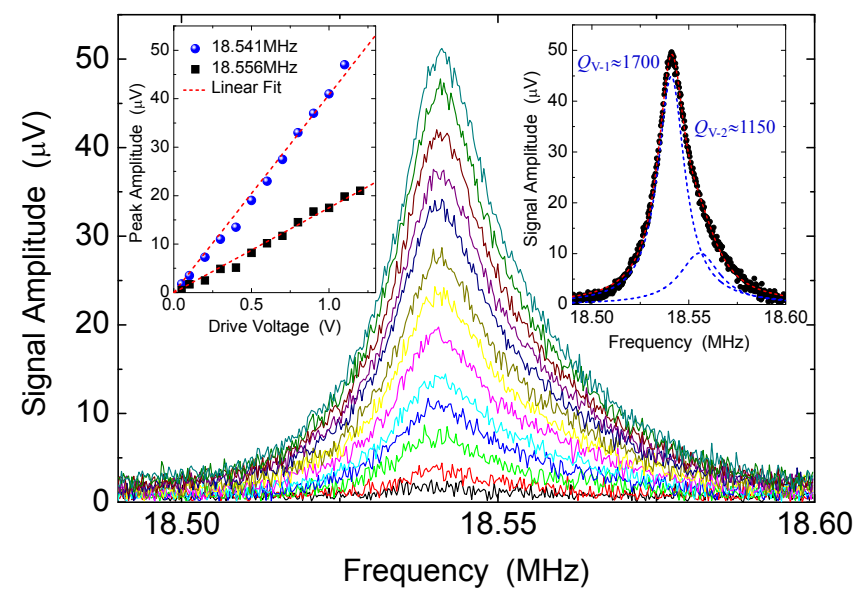

Figure 9: Measured response due to a very uneven pair of split modes at $\sim 18.54 \mathrm{MHz}$ (dominating) \& 18.56MHz. Left Inset: Peak amplitude versus drive with linear fits. Right Inset: Revelation of the contributions from the two split modes, and the extracted $Q$ 's.

\section{CONCLUSIONS}

We have experimentally demonstrated and investigated circular thin-film microdisk resonators nanomachined from a $500 \mathrm{~nm} \mathrm{SiC}$ epilayer grown on $\mathrm{Si}$, which exhibit multi-mode flexural resonances in the range of $\sim 2-20 \mathrm{MHz}$ with typical $Q$ 's 1000-4000. Sensitive interferometric readout and modeling together reveal the characteristics of observed modes, and the effects of varying anchor size upon the frequency spectra and $Q$ 's of the devices. The micromechanical and RF characteristics of these resonators and arrays make them interesting for potential applications including on-chip resonance-based physical sensing elements and multi-mode communication building blocks.

\section{REFERENCES}

[1] R.A. Johnson, Mechanical Filters in Electronics, John Wiley \& Sons, Inc., New York (1983).

[2] M. Rodahl, F. Höök, A. Krozer, P. Brzezinski, B. Kasemo, Rev. Sci. Instrum. 66, 3924-3930 (1995).

[3] J.R. Clark, W.-T. Hsu, C.T.-C. Nguyen, Tech. Digest, IEDM 2000, pp. 493-496, San Francisco, CA, Dec. 11-13 (2000).

[4] C.T.-C. Nguyen, IEEE Trans. Ultrason. Ferroelectr. Freq. Control 54, 251-270 (2007).

[5] Y.T. Yang, C. Callegari, X.L. Feng, M.L. Roukes, Nano Letters 11, 1753-1759 (2011).

[6] X.L. Feng, C.J. White, A. Hajimiri, M.L. Roukes, Nature Nanotechnology 3, 342-346 (2008).

[7] X.L. Feng, C.A. Zorman, M. Mehregany, M.L. Roukes, Tech. Digest, Hilton Head'2006, pp. 86-89, Hilton Head Island, SC, June 4-8 (2006).

[8] X.L. Feng, M.H. Matheny, C.A. Zorman, M. Mehregany, M.L. Roukes, Digest of Tech. Papers, Transducers'2009, pp. 2246-2249, Denver, CO, June 21-25 (2009).

[9] C.A. Zorman, A.J. Fleischman, A.S. Dewa, M. Mehregany, P. Pirouz, et al., J. Appl. Phys. 78, 5136-5138 (1995).

[10] A.C. Barnes, R.C. Roberts, N.C. Tien, C.A. Zorman, P.X.-L. Feng, Digest of Tech. Papers, Transducers'2011, pp. 26142617, Beijing, China, June 5-9 (2011). 